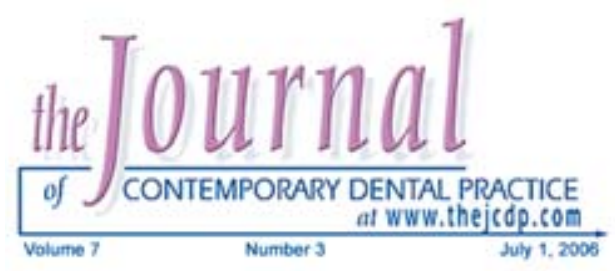

\title{
A 24-Hour Dental Plaque Prevention Study with a Stannous Fluoride Dentifrice Containing Hexametaphosphate
}
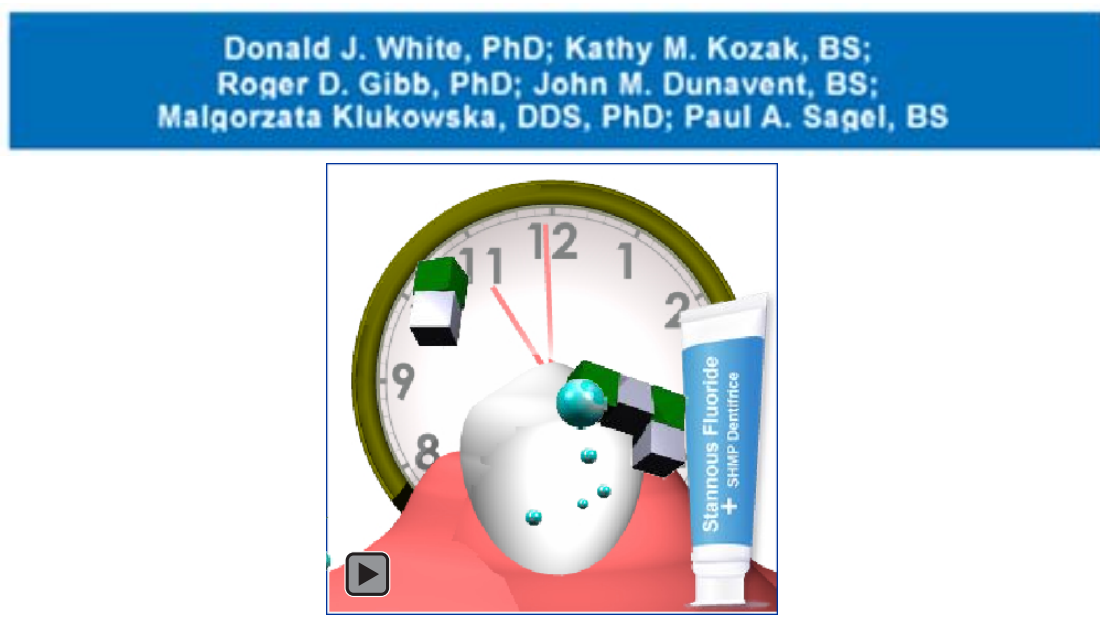

Abstract

Recently, a novel antibacterial fluoride dentifrice containing stannous fluoride and sodium hexametaphosphate (Crest $_{\circledast}$ PRO-HEALTH ${ }^{\mathrm{TM}}$ ) was introduced. A digital plaque image analysis (DPIA) technique was used to quantify in situ plaque formation in a population carrying out a phased intervention protocol that included: (1) an initial treatment regimen including toothbrushing with standard sodium fluoride dentifrice in conventional bid brushing, (2) a second treatment regimen where a modified hygiene regimen was applied using standard sodium fluoride dentifrice including a period of 24 hours of non-brushing, and (3) a third treatment regimen where the 24-hour non-brushing regimen was continued using the antimicrobial stannous fluoride/sodium hexametaphosphate dentifrice. The quantitative evaluation of plaque formation was assessed in morning measurements following either standard evening hygiene (treatment period 1) or 24 hours since brushing (treatment periods 2 and 3). Post-brushing plaque measurements were also taken in each treatment regimen. Sixteen subjects completed all three treatment regimens with no side effects or oral complaints. Morning plaque coverage in treatment period 1 was $13.3 \%$. Plaque coverage significantly increased in treatment period 2 when pre-bedtime brushing was discontinued, with 24-hour growth covering $18.4 \%$ of the dentition. Intervention of the antimicrobial stannous fluoride/hexametaphosphate dentifrice in treatment period 3 provided significant inhibition of plaque regrowth over 24 hours $(15.2 \%$ coverage, a 17\% reduction vs. sodium fluoride dentifrice control). These results support the strong retention and lasting antimicrobial efficacy of high stabilized stannous fluoride/sodium hexametaphosphate dentifrices.

Keywords: Stannous fluoride, sodium hexametaphosphate, plaque, dentifrice, Crest PRO-HEALTH toothpaste, Polyfluorite System

Citation: White DJ, Kozak KM, Gibb RD, Dunavent JM. A 24-Hour Dental Plaque Prevention Study with a Stannous Fluoride Dentifrice Containing Hexametaphosphate. J Contemp Dent Pract 2006 July;(7)3:001-011.

(C) Seer Publishing 


\section{Introduction}

Gingivitis results from the development of microbial dental plaque infection at the gingival tooth interface initiating a host response. ${ }^{1-3}$

While certain microbial populations have been correlated with disease processes, the generalized non-specific correlat ion between plaque and soft tissue disease is supported by the onset of inflammation and gingival bleeding when gingivitis is induced, married with a rapid return to health when hygiene measures are reinstituted. ${ }^{4-5}$ Despite the proven benefits of effective oral hygiene for maintenance of soft tissue health, patients continue to fall short of ideal plaque control. ${ }^{6-7}$ Accordingly, the prevalence of gingivitis remains unacceptably high, reported to affect roughly half of U.S. adults.

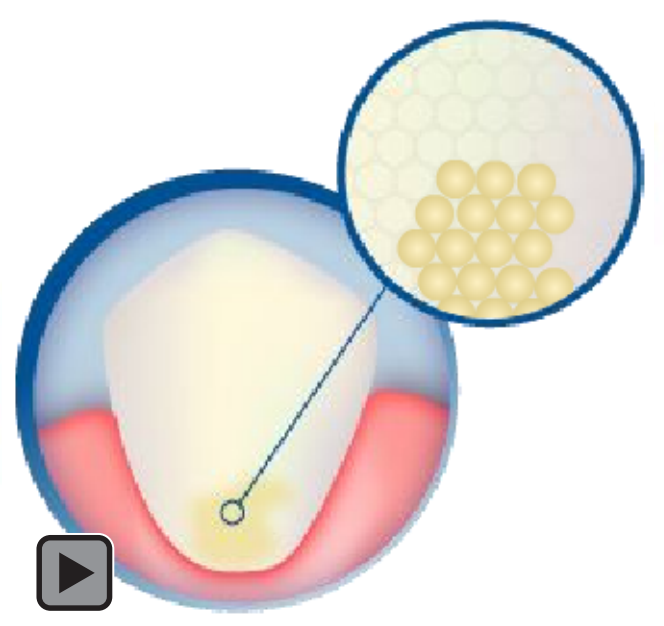

Although most patients find it challenging to achieve complete plaque control with oral hygiene alone, the majority of patients incorporate dentifrice products into their daily hygiene regimens. Due to their frequent application, dentifrices represent opportunistic delivery systems to provide the chemotherapeutic benefits of antibacterial agents. Developing innovative dentifrice and oral rinse formulations with topical antimicrobial ingredients has been a focus area of academic and industry sponsored research. Agents found in commercial oral care products today include chlorhexidine, triclosan, cetylpyridinium chloride, essential oils, and various metal salts including zinc compounds and stannous fluoride..$^{10-15}$ In general oral care formulations with these ingredients have been shown to provide at least some level of antiplaque and antigingivitis efficacy. The benefits provided by these formulations vary and appear to be affected by numerous factors, such as: $^{: 16}$ (1) spectrum of antimicrobial efficacy; (2) adequate bioavailability, demonstrated by plaque penetration and reactivity; and (3) evidence of sustained antimicrobial effects after use.

Stannous fluoride is a common anticaries ingredient which has, more recently, been shown to provide antimicrobial, antiplaque, and antigingivitis efficacy in stabilized or high bioavailable forms. ${ }^{17-19}$ In published clinical studies stannous fluoride dentifrices have been shown to provide significant reductions in plaque (6.9-22.7\%), gingivitis (18-22\%), and gingivial bleeding (27.5-57.1\%) vs. negative controls. ${ }^{17-19}$ A stannous fluoride dentifrice has most recently been reported to be formulated with inclusion of sodium hexametaphosphate as an advanced whitening and calculus agent. This novel dentifrice technology, marketed as Crest $_{\oplus}$ PROHEALTH ${ }^{T M}$ Polyfluorite System, has demonstrated a remarkable combination of clinical benefits including improved effects on gingivitis and gingival bleeding. ${ }^{20-22}$

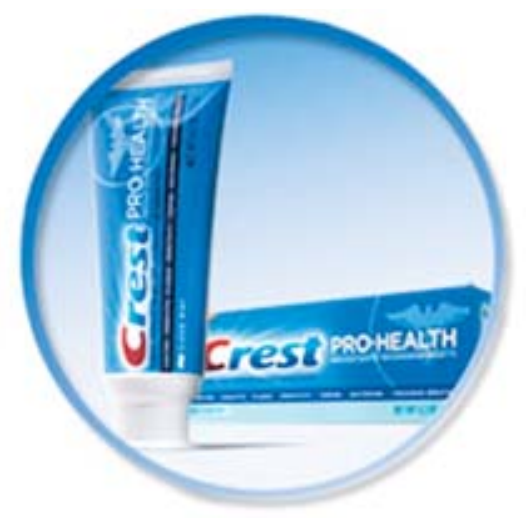

The provision of therapeutically significant gingival health effects by antimicrobials such as stannous fluoride is generally supported by demonstration of tenacious substantivity and activity following use. ${ }^{16}$ In this study the chemotherapeutic substantivity of stannous fluoride is combined with sodium hexametaphosphate. Crest PROHEALTH dentifrice was assessed in a modified brushing regimen model to determine if antiplaque efficacy extended to 24 hours post use. 


\section{Method and Materials}

\section{General Study Design}

This study followed a three treatment-period intervention design with a pre-qualified group of plaque formers. ${ }^{23}$ In treatment period 1, subjects brushed in morning and at night with a sodium fluoride dentifrice (Crest Cavity Protection Regular). During this period, subjects received plaque evaluations both prior and following morning toothbrushing and in afternoons following morning toothbrushing. In treatment period 2, subjects carried out one week use of the sodium fluoride dentifrice following a modified brushing regimen to permit an assessment of sustained antiplaque effects. This was accomplished by 'skipping' a brushing the evening prior to grading days, thereby, forcing a 24 hour period between hygiene and plaque evaluations. Plaque assessments took place both a.m. prebrushing and post-brushing. Treatment period 3 involved one week of toothbrushing with the novel stannous fluoride/hexametaphosphate dentifrice (Crest PRO-HEALTH) following the same modified brushing regimen and plaque assessment schedule used in treatment period 2 to permit an assessment of sustained antiplaque effects. The study logistics are highlighted in Figure 1.

\section{Subject Qualifications}

Subjects entering the protocol were part of a pre-qualified team panel at the Health Care Research Center who participated in previous plaque clinical studies. ${ }^{23}$ The application of the digital plaque image analysis (DPIA) methodology in internal test panels of dentifrices has been reviewed and approved by the Institutional Ethics Review Committee. Subjects signed informed consent prior to participation in this protocol. These subjects exhibited reproducible 'off treatment' plaque levels on teeth both before and after hygiene applications and were trained in new protocols and oral hygiene techniques.

\section{Treatment Sequence}

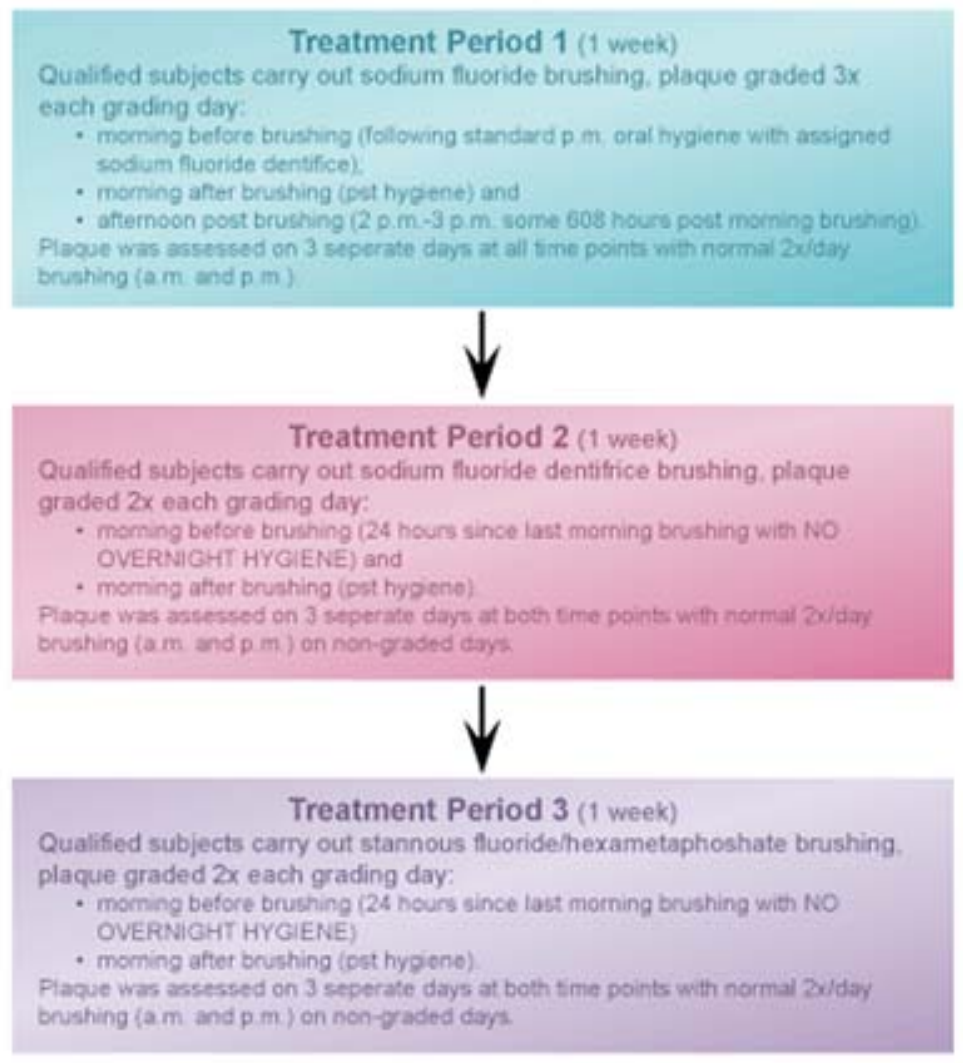

Figure 1. The treatment sequence. 
Subjects were initially screened to ensure:

- Agreement to restricted (no) use of other oral hygiene products while on the study including mouthrinses, toothpastes, etc. Subjects who flossed regularly were allowed to floss between the posterior teeth only throughout the study. Subjects were asked to avoid chewing gum unless it was a daily habit that should be consistently maintained throughout the study.

- No dental visits planned for duration of panel participation.

- No recent (two week) use of antibiotics.

- No special dietary restrictions and/or diet adjustments expected during the trial.

- No 'color matched' restorations on facial surfaces of the anterior teeth.

- Ability to meet all scheduled visits in the protocol and comply with instructions.

- Agreement to refrain from participation on other panels and agreement not to use other oral products than those assigned, including no use of whitening or therapeutic chewing gums or whitening formulations.

- Good general health and good overall dental health (as self assessed and confirmed by subject disclosure confirming routine dental visits).

- No significant general allergy problems or specific allergies to dyes.

- Sufficient plaque levels in pilot prescreening to warrant participation.

- No pregnancy or nursing.

- No routine adverse reactions to currently marketed oral care products.

- Test Products

The assigned toothpaste for treatment periods 1 and 2 was standard Crest Cavity Protection Regular Dentifrice (NaF, silica abrasive, Regular flavor, The Procter \& Gamble Co., Cincinnati, $\mathrm{OH}$, USA). This dentifrice contains sodium fluoride at FDA monograph levels for cavity protection, standard abrasives, conventional levels of surfactant and preservatives, and no specialized tartar control or antimicrobial ingredients. Accordingly, use of this dentifrice could be considered to provide a more-or-less standard of hygiene care. The dentifrice was supplied in overtubed (blind) commercial packaging with study labeling containing instructions. The assigned dentifrice for treatment period 3 was
Crest PRO-HEALTH, comprised of $0.454 \%$ stannous fluoride complemented with sodium hexametaphosphate (antitartar and whitening ingredient) and silica. ${ }^{24}$ This dentifrice was supplied in generic tubes with study labeling only. Toothbrushes assigned for home use were Oral-B 40 standard brushes (Oral-B, The Procter \& Gamble Company, Cincinnati, OH, USA). For supervised clinic brushing, subjects were provided disposable manual test brushes.

\section{Detailed Treatment Protocols}

Treatment Period 1: Subjects entered the dental panel clinic on a Friday and were provided with the sodium fluoride dentifrice and an OralB 40 Soft toothbrush. Subjects were instructed to brush as they normally do twice per day throughout the weekend with typical timings prior to bed and in the morning on rising. During the week, subjects were graded on three separate occasions (Monday - Friday) permitting off days. On the evening prior to a grading day, subjects carried out their standard p.m. hygiene immediately prior to retiring. Subjects were instructed not to eat or drink after this hygiene. Subjects reported to the imaging laboratory in the 'grading morning' prior to any food/beverages and without oral hygiene. In the imaging clinic, subjects disclosed dental plaque and subjected themselves to a "pre-brush a.m. plaque imaging." Subjects then brushed for 40 seconds with sodium fluoride dentifrice provided in metered 1.5 gram doses on a disposable brush. Following brushing, subjects redisclosed dental plaque and subjected themselves to a second plaque imaging, a so-called "a.m. post-brushing plaque imaging." Subjects were then free to have breakfast and lunch, as well as snacks throughout the grading day. During phase I subjects also reported to the imaging clinic for a p.m. plaque regrowth measure, as customary for this protocol, though this measure could not obviously be carried through for the 24 hour assessments in later periods. Subjects were asked to avoid food and drink for at least $1 / 2$ hour prior to this evaluation. Subjects participated in three replicate visits with three measures taken each day during this and each subsequent treatment period (see Figure 2).

Treatment Period 2: Subjects entered the dental panel clinic and were provided with a new supply of sodium fluoride dentifrice, an Oral-B 40 Soft 


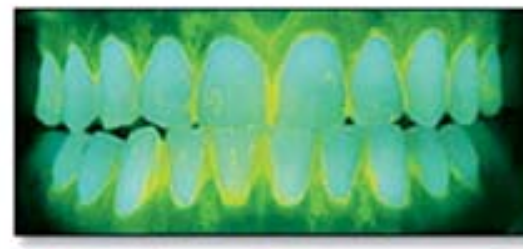

\section{Pre-brushing Image}

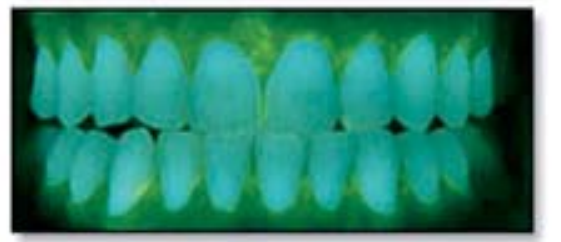

Post-brushing Image

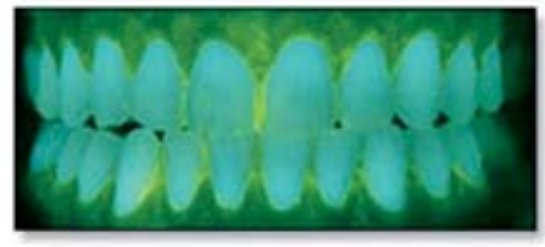

Afternoon Regrowth Image

Figure 2. Typical images as screened in DPIA disclosure and objective measures. The images show the variations in plaque response seen in DPIA imaging -including pre-brushing, post brushing, and p.m. imaging encountered in this disclosure and grading technique.

toothbrush, and new hygiene study instructions. Subjects were instructed to brush as they normally do twice per day throughout the weekend with typical timings prior to bed and in the morning on rising. During the week, subjects were graded on three separate occasions (Monday - Friday) permitting off days. On the day prior to a grading day, subjects carried out their standard a.m. hygiene prior to breakfast. Subjects were instructed to eat and drink as normal throughout the day but to carry out NO P.M. HYGIENE prior to bed in the evening. Subjects were instructed to report to the imaging laboratory in the 'grading morning' prior to any food/beverages and without oral hygiene. Thus, 24 hours had elapsed since subjects had carried out oral hygiene. Subjects reported to the imaging clinical for plaque measurement following the same procedure described for treatment period 1.

Treatment Period 3: The treatment and imaging procedure followed in this treatment period was identical to that of treatment period 2 except subjects used a $0.454 \%$ stannous fluoride and hexametaphosphate dentifrice instead of a sodium fluoride dentifrice.

\section{Dental Plaque Assessments}

\section{Evaluation Parameters}

Dental plaque coverage was analyzed by a standardized digital imaging protocol previously described in detail. ${ }^{25}$ The key steps of this method are illustrated in the corresponding video clip.

\section{Digital Plaque Imaging System}

(Go to the online article to view this clip.)

Digital imaging included the capture of UV images of disclosed plaque in subjects. Plaque was illuminated using two Balcar long wave UV flashes (model FX60) equipped with cutoff filters at $265 \mathrm{~nm}$. The flashes were powered by two Balcar 2400 power packs and controlled by the computer image capture system. The flashes were positioned at 45 degree angles to the subject to reduce reflection to a minimum. To protect the subject's eyes, UV filtering glasses were worn on 


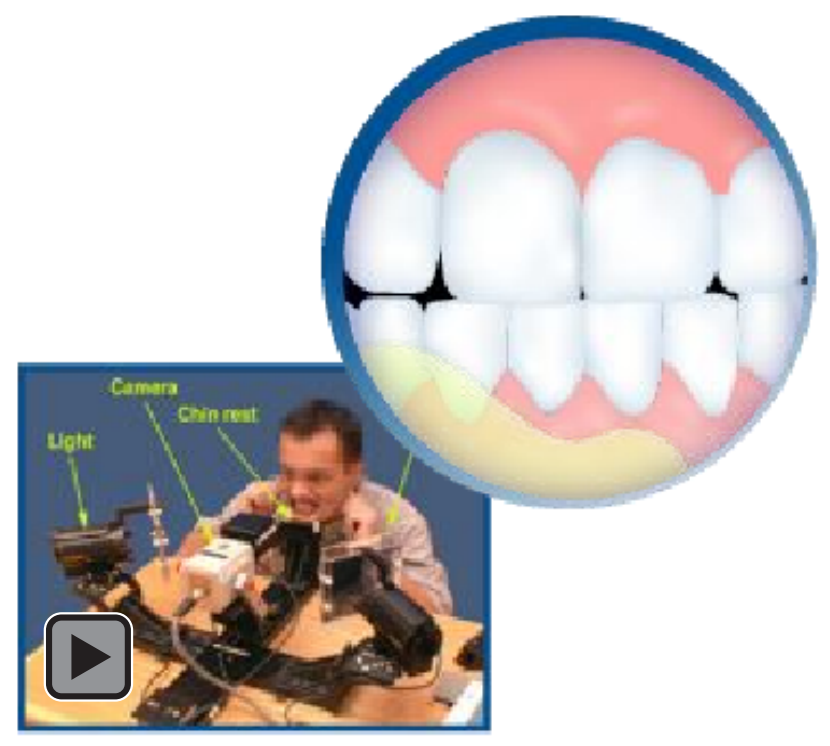

imaging or, for experienced subjects, instructions were given to close the eyes during image capture. UV images were captured with a Fuji 1000 CCD camera controlled by a desktop computer. For plaque measures, subjects sat in front of the camera and positioned their facial dentition in a specialized chin rest at an identical distance of $45.5 \mathrm{~cm}$ from the image capture camera. Lip retractors were used by the subjects, who, after training, could position themselves for imaging to allow uniform lighting of the teeth and to capture unobstructed images. To ensure good subject positioning a live image was displayed on a positioning monitor. Once a good position was established, a UV image was captured and a reference position image was stored according to a subject identification number.

The imaging system was calibrated using standard Munsell color charts with red, green and blue (RGB) values corrected $(<5 \%)$ for system stability in standardized assessments. Captured images were analyzed and classified with Optimas $\mathrm{R}$ macros. Discriminant analysis was used to statistically classify pixels into different anatomical categories, e.g., teeth, gums, plaque on gums, clean teeth, clean gums, etc. Total image pixels were collected and assigned to respective designations. The most reproducible analysis typically includes the ratio of plaque on teeth pixels to total teeth pixels (clean teeth + plaque covered teeth); this representing an 'area' coverage estimate for each image, and this analysis was carried out herein. The DPIA technique provides a number of advantages to categorical indices. The method is quite rapid, holistic on the facial dentition, and quantitative. Importantly, it easily is adapted to multiple diurnal grades and in repeated measures. The digital imaging approach to objective plaque determination is supported by proportional rates of plaque regrowth in lingual, facial, maxillary, and mandibular dentition areas. ${ }^{26-28}$ The computer designated plaque area is used to calculate a percentage dentition coverage estimate for the facial plaque image, which was then compared for treatment effects. It is noteworthy that operator/analyst contribution to study measures only takes place in standardization of custom analysis rules for pixel classifications of 'plaque covered tooth' or 'plaque free tooth,' etc. Once quantitative decision rules are established, plaque assessment is quantitatively managed by the computer decision rule, and no subjective clinician grading is involved. In this context then, the DPIA is operator independent and no subjective clinician effects are permitted or expected.

Plaque disclosure for imaging utilized fluorescein buffer solution containing 1240 ppm fluorescein. Prior to photographing, subject plaque is disclosed by fluorescein as follows:

1. Rinse for 10 seconds with $25 \mathrm{ml}$ of phosphate buffer

2. Rinse for 1 minute with $5.0 \mathrm{ml}$ of $1240 \mathrm{ppm}$ fluorescein in phosphate buffer;

3. Rinse $3 \times 10$ seconds with $25 \mathrm{ml}$ of phosphate buffer

Phosphate buffer was comprised of 3.62 grams of monosodium phosphate and 0.349 grams of disodium phosphate diluted to 2 liters with ultrapure water. The final $\mathrm{pH}$ of this mixture is 5.5. The solution was prepared fresh each day.

\section{Statistical Analysis}

The efficacy endpoint in this study was the percent of facial tooth surfaces covered with plaque. The efficacy analysis consisted of averaging on a per subject basis the three days of plaque coverage data in each treatment period. Pre-brushing and post-brushing plaque scores were analyzed separately. A paired difference t-test was used to compare the average plaque coverage between treatment periods. All statistical tests were two-sided and performed at the $5 \%$ significance level. 
Table 1. Demographics.

\begin{tabular}{|l|c|}
\hline \multicolumn{2}{|c|}{ Demographlc Characteristic } \\
\hline Age (Years) & \\
\hline Mean & 33.25 \\
\hline Minimum-Maximum & $24-38$ \\
\hline Gender ${ }^{\text {a }}$ & \\
\hline Female & 10 \\
\hline Male & 6 \\
\hline Ethnicity ${ }^{\text {a }}$ & \\
\hline Caucasian & 12 \\
\hline Black & 2 \\
\hline Asian & 2 \\
\hline
\end{tabular}

$a_{=n u m b e r}$ of subjects

Table 2. Morning pre-brushing treatment comparisons.

\begin{tabular}{|l|c|c|c|}
\hline Dentifrice Treatment & Number of Subjects & $\begin{array}{c}\text { Plaque \% Coverage } \\
\text { Mean (SD) }\end{array}$ & $\begin{array}{c}\text { Treatment } \\
\text { Comparison } \\
\text { p-value }\end{array}$ \\
$\begin{array}{l}\text { Sodium Fluoride } \\
\text { 24 Hour Protocol }\end{array}$ \\
\hline $\begin{array}{l}\text { Period 1 - Standard Protocol } \\
\text { Sodium Fluoride }\end{array}$ & 16 & $13.3(4.27)$ & $<0.0001$ \\
\hline $\begin{array}{l}\text { Period 2 - 24 Hour Protocol } \\
\text { Sodium Fluoride }\end{array}$ & 16 & $18.4(5.97)$ & \\
\hline $\begin{array}{l}\text { Period 3 - 24 Hour Protocol } \\
\text { Stannous } \\
\text { Fluoride/Hexametaphosphate }\end{array}$ & 16 & $15.2(6.87)$ & \\
\hline
\end{tabular}

a Two-sided $p$-values from a paired-difference t-test.

\section{Results}

Sixteen subjects completed the panel, with no dropouts or side effects reported. Demographic information is reported in Table 1. There were ten women and six men. Subjects ranged in age from 24 to 38 years. The majority of subjects (75\%) were Caucasian.

Table 2 shows the results of the efficacy analysis for morning pre-brushing plaque evaluations. The table shows modification of oral hygiene with the sodium fluoride dentifrice in the form of 'skipping' an evening oral hygiene resulted in a $38 \%$ increase in plaque coverage on teeth, with average plaque coverage increasing from
$13.3 \%$ to $18.4 \%$. This difference was highly statistically significant $(p<0.0001)$. The intervention of the stannous fluoride/sodium hexametaphosphate dentifrice within the 24 hour protocol produced a $17.4 \%$ reduction in a.m. prebrushing plaque coverage in subjects compared to like brushing with the sodium fluoride dentifrice. This difference was also highly statistically significant $(p=0.0002)$.

Table 3 highlights results of the plaque analysis for morning post-brushing plaque evaluations. The table shows morning toothbrushing with the sodium fluoride dentifrice resulted in generally similar levels of dentition cleaning regardless of 
Table 3. Post-brushing treatment comparisons.

\begin{tabular}{|l|c|c|c|}
\hline Treatment & Number of Subjects & $\begin{array}{c}\text { Plaque \% Coverage } \\
\text { Mean (SD) }\end{array}$ & $\begin{array}{c}\text { Treatment } \\
\text { Comparison } \\
\text { p-value } \\
\text { Sodlum } \text { viuoride } \\
\text { 24 Hour Protocol }\end{array}$ \\
\hline $\begin{array}{l}\text { Period 1 - Standard Protocol } \\
\text { Sodium Fluoride }\end{array}$ & 16 & $6.4(1.80)$ & 0.11 \\
\hline $\begin{array}{l}\text { Period 2 - 24 Hour Protocol } \\
\text { Sodium Fluoride }\end{array}$ & 16 & $7.3(3.64)$ & \\
\hline $\begin{array}{l}\text { Period 3 - 24 Hour Protocol } \\
\text { Stannous } \\
\text { Fluoride/Hexametaphosphate }\end{array}$ & 16 & $6.8(3.52)$ & 0.15 \\
\hline
\end{tabular}

a Two-sided $p$-values from a paired-difference t-test.

overnight hygiene regimen. Thus, the post brushing plaque coverage measured $7.3 \%$ in subjects who did not carry out hygiene for 24 hours and $6.4 \%$ in subjects who carried out normal p.m. hygiene. These differences were not statistically significant. Likewise, subjects who brushed with stannous fluoride/sodium hexametaphosphate dentifrice presented with $6.8 \%$ plaque coverage on dentition, numerically similar and not statistically significantly different from post brushing evaluations while using sodium fluoride dentifrice with either hygiene regimen. (In all cases, the act of morning toothbrushing produced statistically significant $(p<0.05)$ reductions in plaque coverage on dentition, as expected.)

To assess the repeatability of plaque formation for the study population and the validity of a phased intervention treatment design, we compared plaque outcomes across repeated measures. Specifically, morning pre- and post-brushing mean plaque coverage was summarized separately for each of the three imaging visits per treatment period. It is clear from Figures 3 and 4 there was no consistent increasing or decreasing trend within treatment period, suggesting panelists had come to a plaque formation steady state.

A repeated measures analysis was also performed to compare the means between visits by treatment period and no statistically significant differences were found at the $10 \%$ significance level.

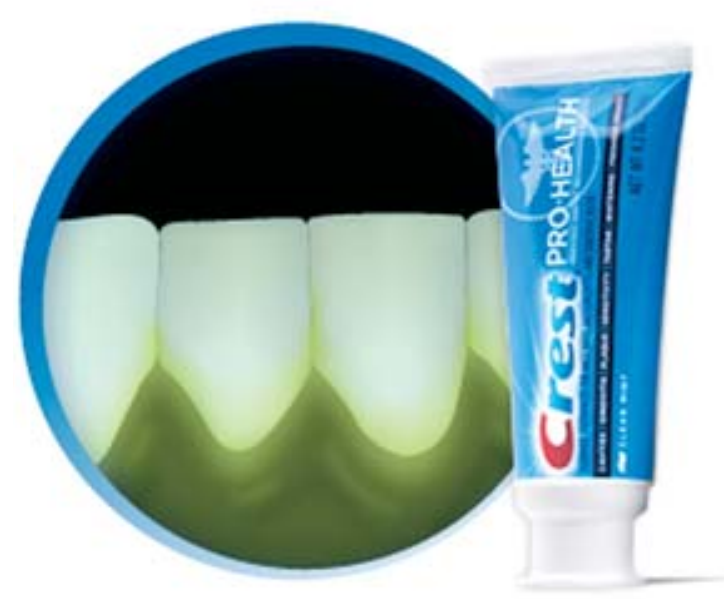

\section{Discussion}

The purpose of this study was to assess the effect of a novel stannous fluoride/sodium hexametaphosphate dentifrice in the prevention of dental plaque formation for 24 hours following brushing as compared to like hygiene pattern using standard sodium fluoride dentifrice. Results show the use of stannous fluoride/sodium hexametaphosphate dentifrice by subjects produced a statistically significant reduction in dental plaque coverage of their dentition 24 hours following the last use, as compared to like use of the sodium fluoride dentifrice. Although brushing is directed for use bid, these results show patients may expect up to 24 hours sustained protection following use of stannous fluoride/sodium hexametaphosphate dentifrice as compared to like hygiene pattern using standard sodium fluoride dentifrice. Importantly, these results speak generally to the substantivity of 


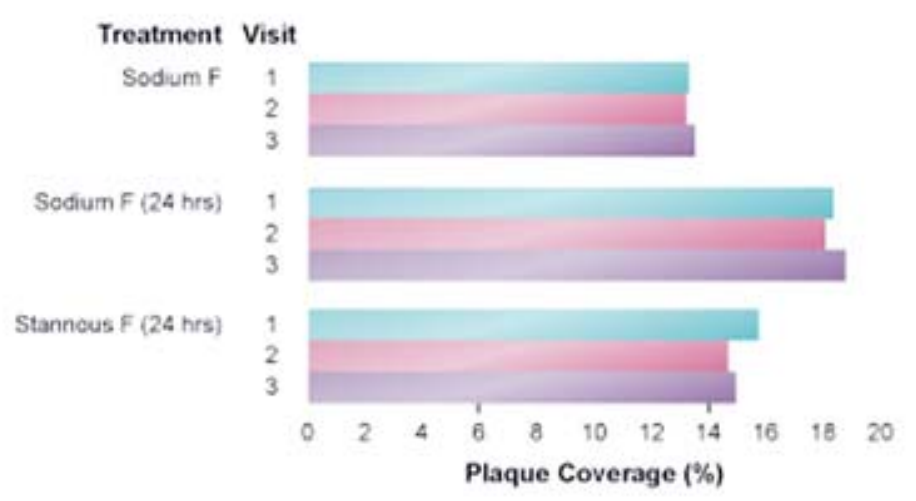

Figure 3. Pre-brushing plaque coverage repeatability.

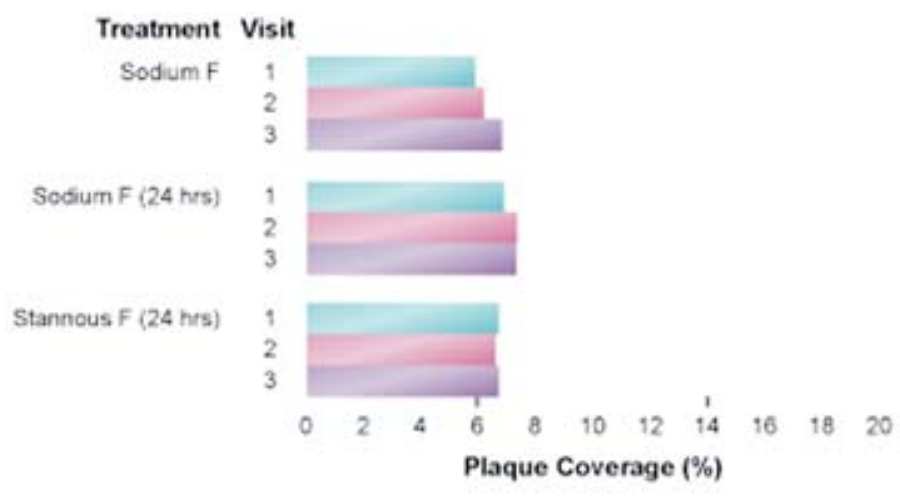

Figure 4. Post-brushing plaque coverage repeatability.

Crest PRO-HEALTH dentifrice effects in inhibiting plaque formation, although usage patterns of dentifrice by consumers varies widely. Supporting data for application of the repeated measures DPIA intervention protocol design can be seen in Figures 3 and 4, where replicate measures of p.m. plaque growth and post brushing plaque are observed to remain stable throughout treatment period 1 . Stability of plaque while using control dentifrice provides some assurance that effects seen on intervention to new treatment are the result of treatment effects and not panel drift. The demonstration of statistically significant benefits in plaque removal with toothbrushing provides additional internal standardization of each DPIA test protocol.

The capability to compare both the extent and duration of antiplaque effects - including both pre- and post hygiene periods in subjects with typical levels of plaque accumulation - are important benefits of the DPIA technique. The use of fluorescence imaging permits the non invasive quantitative assessment of plaque along the gumline, in regions often graded by gingival probing in conventional clinician applied categorical indices. The grading of these marginal plaque levels, in subjects with normal hygiene, is logistically complicated in conventional techniques and, hence, limits their use in the fashion employed herein. As a result, the DPIA technique is able to assess the prevention of plaque formation and benefits of plaque removal throughout the day, providing a quantitative means for the assessment of not only the magnitude but the duration of antiplaque efficacy. Importantly, this grading sensitivity is realized without subjective assessments but with standardized decision rules independent of clinician influences.

The supragingival plaque reductions provided by use of stannous fluoride/sodium hexametaphosphate dentifrice likely contributes to 
the proven therapeutic efficacy of this formulation in reducing gingivitis and gingival bleeding. ${ }^{20-}$

${ }^{22}$ The chemotherapeutic and hygiene directed control of dental plaque is generally considered as useful preventive measures in arresting and reversing progressive periodontal disease over time in patients. ${ }^{29,30}$ Supragingival plaque control can reduce the progression and manifestations of chronic gingivitis because: (a) suppression of overall bacterial load may reduce gingival insult due to specific pathogens or bacterial metabolites; (b) reduced area coverage of plaque is naturally associated with thinner plaques, which may have reduced potential for growth of typically anaerobic periodontal pathogens; (c) reduced gingival inflammation results in better gingival-tooth architecture - limiting the trapping of periodontal pathogens and permitting better hygiene; and (d) reduced inflammation can limit the production of gingival exudate - again limiting the expression and proliferation of periodontal pathogens that thrive in the inflammatory environment.

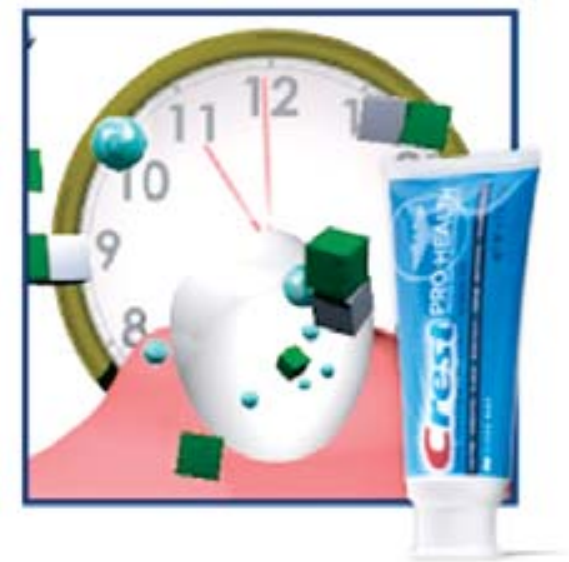

\section{Conclusion}

Using the DPIA technique, this research shows the novel dentifrice with stabilized stannous fluoride and sodium hexametaphosphate dentifrice inhibits plaque regrowth over a 24-hour period significantly better than a standard sodium fluoride dentifrice.

\section{References}

1. Robinson PJ. Gingivitis: A Prelude to Periodontitis? J Clin Dent. 1995;6:41-45.

2. Mombelli AW. The role of dental plaque in the initiation and progression of periodontal diseases. In: Lang NP, Attstrom R, Loe H. Proceedings of the European Workshop on Mechanical Plaque Control. Berlin: Quintessenz Verlag, 1998;85-97.

3. Shibly O, Rifai S, Zambon JJ. Supragingival plaque in the etiology of oral diseases. Periodontol 2000. 1995;8:42-59.

4. Loe H, Theilade E, Jensen SB. Experimental gingivitis in man. J Periodontol. 1965;36:177-187.

5. Theilade $\mathrm{E}$, Wright $\mathrm{WH}$, Jensen $\mathrm{BS}$, Loe H. Experimental gingivitis in man II. A longitudinal clinical and bacteriological investigation. J Periodontol Res. 1966;1:1-13.

6. Schou L. Behavioral aspects of dental plaque control measures: An oral health promotion perspective. In: Lang NP, Attstrom R, Loe H. Proceedings of the European Workshop on Mechanical Plaque Control. Berlin: Quintessenz Verlag, 1998; 287-299. (1998).

7. Tedesco LA. Behavioral research related to oral hygiene practices: a new century model of oral health promotion. Periodontol 2000. 1995;8:15-23.

8. Baehni PC, Bougeois DM. Epidemiology of periodontal health and Inglehart M, disease. In: Lang NP, Attstrom R, Loe H. Proceedings of the European Workshop on Mechanical Plaque Control. Berlin: Quintessenz Verlag, 1998; 19-34.

9. National Center for Health Statistics. Healthy People 2000 Review, 1998-99. Hyattsville, Maryland: Public Health Service, 1999. Library of Congress Catalog Card \# 76-641496.

10. Van der Ouderaa FJG. Human clinical studies of antiplaque and antigingivitis agents dosed from a dentifrice. In: Rolla G, Embery G. Clinical and Biological Aspects of Dentifrices, Oxford, Oxford University Press, 1992;181-204.

11. Cummins D, Creeth JE. Delivery of antiplaque agents from dentifrices, gels and mouthwashes. J Dent Res. 1992;68 (special issue): 1609-1616.

12. Ciancio SG. Agents for the management of plaque and gingivitis. J Dent Res. 1992;71:1450-1454.

13. Davies RM, Ellwood RP, Davies GM. The effectiveness of a toothpaste containing Triclosan and polyvinyl-methyl ether maleic acid copolymer in improving plaque control and gingival health. J Clin Periodontol. 2004;31:1029-1033. 
14. Jackson RJ. Metal salts, essential oils and phenols - old or new? Periodontol 2000. 2002;15:63-73.

15. White DJ. A "Return" to stannous fluoride dentifrices. J Clin Dent. 1995;6:29-36.

16. Fine DJ. Periodontol 2000. 1995;8:87-107.

17. Beiswanger BB, Doyle PM, Jackson RD, Mallatt, ME, Mau MS. The clinical effect of dentifrices containing stabilized stannous fluoride on plaque formation and gingivitis - a six month study with ad libitum brushing. J Clin Dent. 1995;6 (Special Issue): 46-53.

18. Perlich MA, Bacca LA, Bollmer BW, Lanzalaco AC, McClanahan SF, Sewak LK, Beiswanger BB, Eichold WA, Hull JR, Jackson RD, Mau MS. The clinical effect of a stabilized stannous fluoride dentifrice on plaque formation, gingivitis and gingival bleeding: a six month study. J Clin Dent. 1995; 6 (Special Issue):54-58.

19. Beiswanger BB, McClanahan SF, Bartizek RD, Lanzalaco AC, Bacca LA, White DJ. The comparative efficacy of stabilized stannous fluoride dentifrice, peroxide/baking soda dentifrice and essential oil mouthrinse for the prevention of gingivitis. J Clin Dent. 1997; 8:46-53.

20. Archila L, He T, Winston JL, Biesbrock AR, McClanahan SF, Bartizek RD. Antigingivitis efficacy of a stabilized stannous fluoride/sodium hexametaphosphate dentifrice in subjects previously nonresponsive to a triclosan copolymer dentifrice. Compend Contin Educ Dent. 2005;26:12-18.

21. Archila L, Bartizek RD, Winston JL, Biesbrock AR, McClanahan SF, He T. The comparative efficacy of stabilized stannous fluoride/sodium hexametaphosphate dentifrice and sodium fluoride/triclosan/ copolymer dentifrice for the control of gingivitis: a 6-month randomized clinical study. J Periodontol. 2004 Dec;75(12):1592-9.

22. Mankodi S, Bartizek RD, Winston JL, Biesbsrock AR, McClanahan SF, He T. Anti-gingivitis efficacy of a stabilized $0.454 \%$ stannous fluoride/sodium hexametaphosphate dentifrice: a controlled sixmonth clinical trial. J Clin Periodontol. 2005;32:75-80.

23. Kozak KM, Gibb R, Dunavent J, White DJ. Efficacy of a high bioavailable cetylpyridinium chloride mouthrinse over a 24-hour period: a plaque imaging study. Am J Dent. 2005 Jul;18 Spec No:18A23A.

24. Baig A, He T. A novel dentifrice technology for advanced oral health protection: a review of technical and clinical data. Compend Contin Educ Dent. 2005;26:4-11.

25. Sagel PA, Lapujade PG, Miller JM, Sunberg RJ. Objective quantification of plaque using digital image analysis. In Faller RV Assessment of Oral Health - Diagnostic Techniques and Validation Criteria, Basel, Karger;2000;130-143.

26. Cumming BR, Loe $\mathrm{H}$. Consistency of plaque distribution in individuals without special home care instruction. J Perio Res. 1973; 8: 94-100.

27. Lang NP, Cumming BR, Loe $\mathrm{H}$. Toothbrushing frequency as it relates to plaque development and gingival health. J Periodontol. 1973; 44: 396-405.

28. Straub AM, Salvi GE, Lang NP. Supragingival plaque formation in the human dentition. In: Lang NP, Attstrom R, Loe H. Proceedings of the European Workshop on Mechanical Plaque Control. Berlin: Quintessenz Verlag, 1998; 72-84.

29. Cancro LP, Fischman SL. The expected effect on oral health of dental plaque control through mechanical removal. Periodontol 2000. 1995;8:60-74.

30. Garmyn P, van Steenberghe D, Quirynen M. Efficacy of plaque control in the maintenance of gingival health: Plaque control in primary and secondary prevention. In: Lang NP, Attstrom R, Loe $\mathrm{H}$. Proceedings of the European Workshop on Mechanical Plaque Control. Berlin: Quintessenz Verlag, 1998;107-120. 

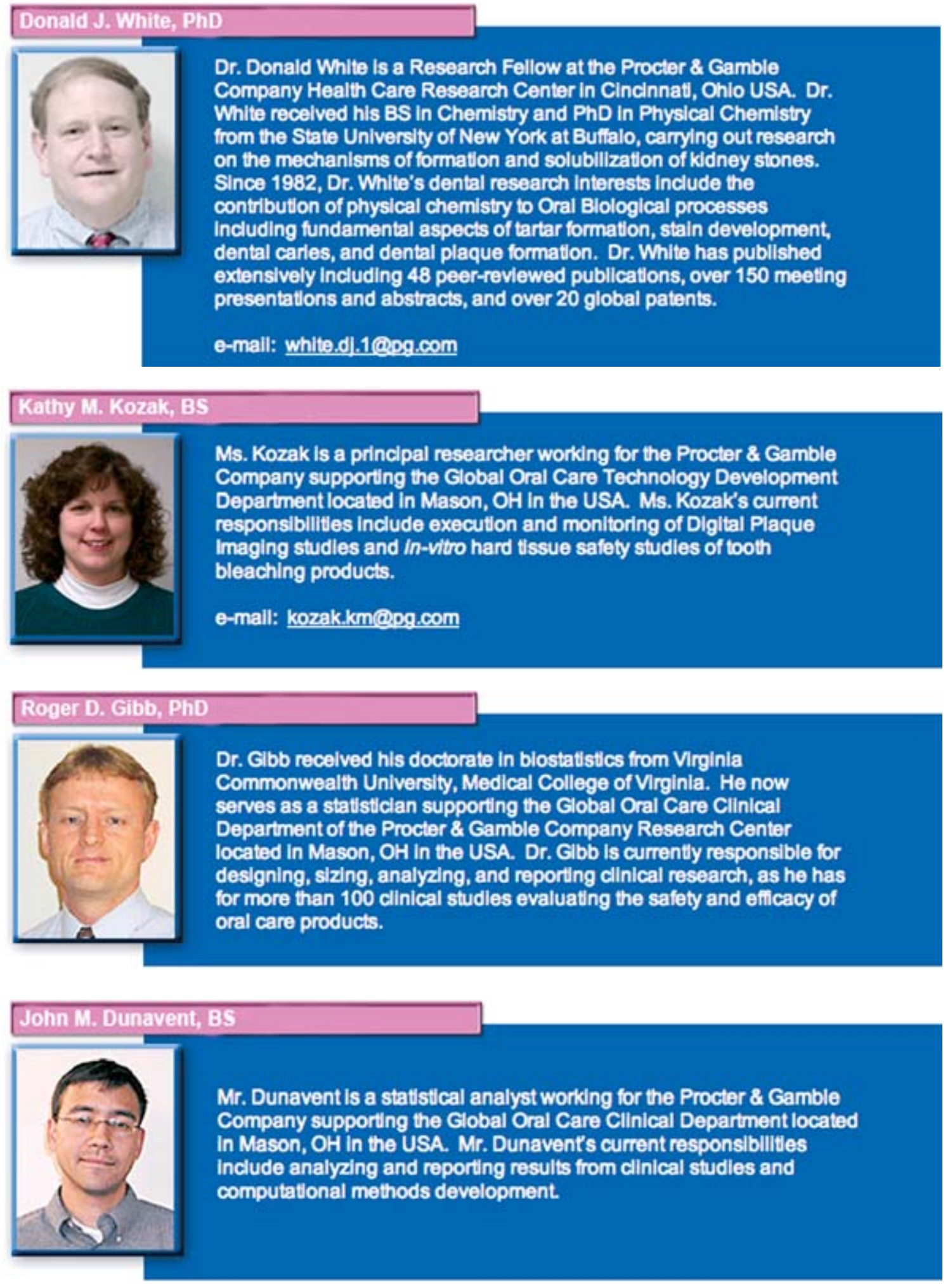

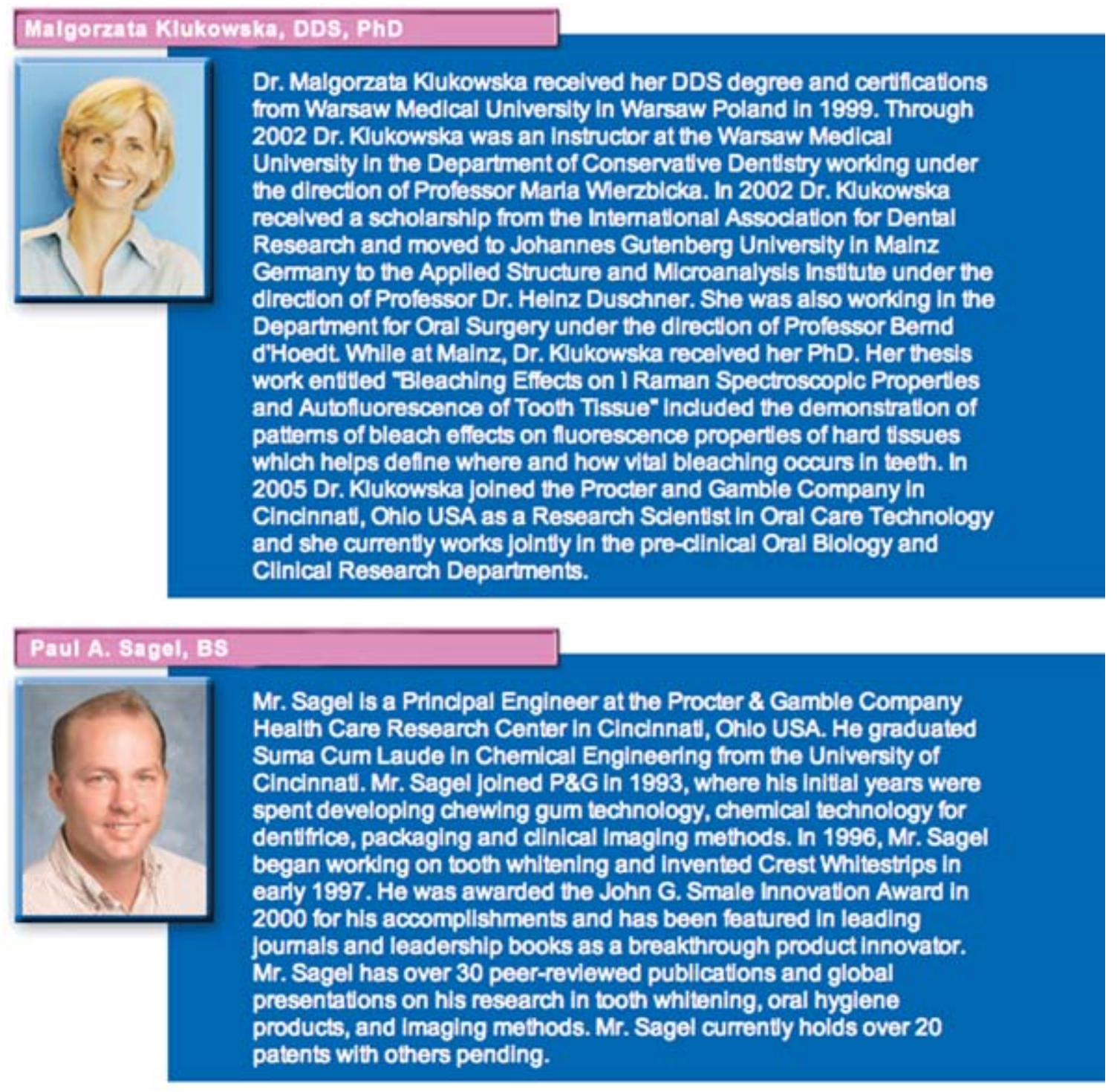

\section{Acknowledgements}

The authors would like to recognize the contributions of Malgorzata Klukowska, Erinn Conde, Paul Sagel and Lisa Sagel to the digital plaque imaging film. 